\title{
INTIMAL-TYPE PRIMARY SARCOMA OF THE THORACIC AORTA PRESENTING AS A SACCULAR FALSE ANEURYSM: REPORT OF A CASE WITH EVIDENCE OF RHABDOMYOSARCOMATOUS DIFFERENTIATION
}

Eugenio Neri, MD, ${ }^{\mathrm{a}}$ Clelia Miracco, MD, ${ }^{\mathrm{b}}$ Pietro Luzi, MD, ${ }^{\mathrm{b}}$ Enrico Carone, MD, ${ }^{\mathrm{a}}$ Alberto Tripodi, MD, ${ }^{\mathrm{a}}$ and Carlo Sassi, MD, Siena, Italy

Primary sarcomas rarely occur in the great vessels. The aorta is a rare site of sarcomas, which mainly involve its abdominal tract. They have been observed more frequently in the pulmonary artery, followed by the inferior vena cava. In the aorta the most common histologic type has been reported as poorly differentiated (so called "intimal") sarcoma. We here report a case of an intimal sarcoma, with rhabdomyosarcomatous differentiation, presenting as a saccular false aneurysm of the thoracic aorta.

Clinical summary. A 68-year-old man with recurrent chest pain and voice disturbances was admitted to our institution. Medical history was significant only for mild hypertension. His general condition was good and symptoms of fever, weight loss, or anorexia were not reported. Intermittent chest pain, which had begun 8 months earlier with an acute and rapidly regressing back pain, was of moderate intensity and was correlated to efforts during heavy physical activities. One week before hospital admission, the onset of hoarseness induced him to consult an otolaryngologist. Direct laryngoscopy demonstrated left vocal cord paralysis resulting from left recurrent laryngeal nerve palsy as confirmed by laryngeal electromyography. Chest film and contrast computed tomographic scan were also performed. A chest radiograph showed an enlargement of the upper descending thoracic aorta, consistent with an aneurysm; there was no evidence of lung tumor. Computed tomography showed a saccular aneurysm located in the lateral aspect of the aortic wall, at the junction between the arch and the descending aorta (Fig 1). The remainder of the aorta exhibited mild atherosclerosis, with diffuse calcifications in the thoracic and abdominal tracts. Regional lymph nodes were not diseased. The patient was therefore admitted to our institution for surgical treatment and scheduled for an urgent operation.

The chest was entered through a posterolateral incision in the bed of the fifth rib, and femoral cardiopulmonary bypass was then instituted. Systemic cooling was initiated immedi-

From the Department of Thoracic and Cardiovascular Surgery and the Institute of Pathological Anatomy and Histology, ${ }^{\mathrm{b}}$ University of Siena, Siena, Italy.

Received for publication March 12, 1999; accepted for publication April 12, 1999

Address for reprints: Eugenio Neri, MD, Istituto di Chirurgia Toracica e Cardiovascolare Universitá degli Studi di Siena, Policlinico le Scotte, Viale M. Bracci, 53100 Siena, Italy.

J Thorac Cardiovasc Surg 1999;118:371-2

Copyright (C) 1999 by Mosby, Inc.

$0022-5223 / 99 \$ 8.00+0 \quad \mathbf{1 2 / 5 4 / 9 9 2 3 0}$

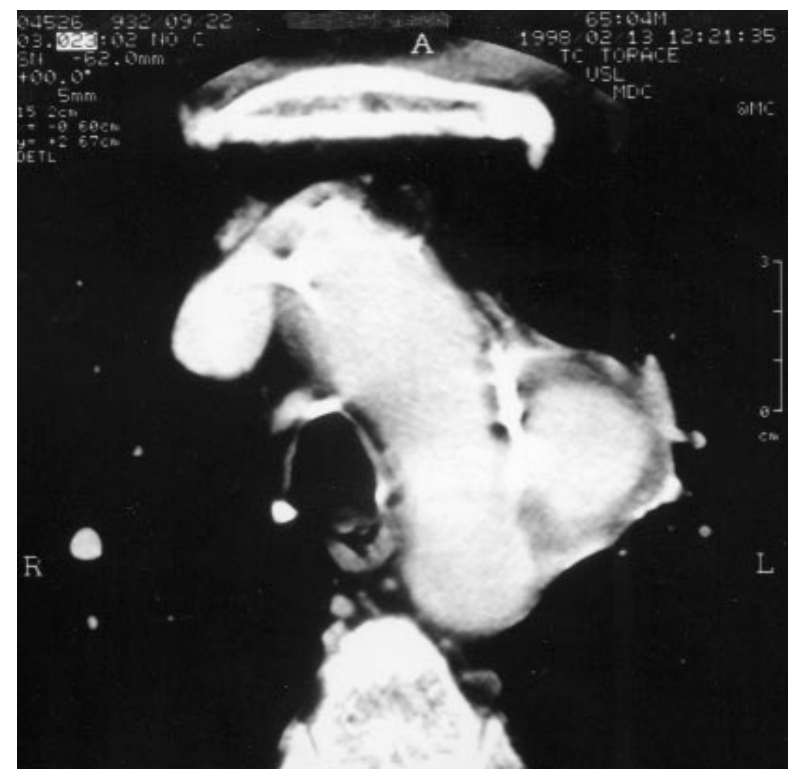

Fig 1. Contrast computed tomographic scan showing an aortic saccular aneurysm located in the lateral aspect of the aortic wall.

ately and deep hypothermic circulatory arrest was obtained at a nasopharyngeal temperature of $20^{\circ} \mathrm{C}$. The saccular aneurysm was opened. The lesion was identified by a perforation of the aortic wall measuring approximately $2.5 \times 3 \mathrm{~cm}$. The edges of the perforation, adequately trimmed, allowed Dacron patch repair. The procedure was completed after 11 minutes of circulatory arrest. The postoperative course was uneventful.

The specimen of the aneurysm wall was histologically examined, and a sarcoma with a rhabdomyosarcomatous differentiation was surprisingly diagnosed. Pathologic findings on the resected specimen, which measured about $4 \mathrm{~cm}$ in its greater diameter, included a solid polyploid vegetation covered with thrombotic substance projecting into the aortic lumen; the remaining aortic wall was slightly thicker than normal, with a few uncomplicated atherosclerotic plaques. On histologic examination, a highly undifferentiated, pleomorphic sarcoma was observed (Fig 2), showing positivity for vimentin, muscle-specific actin HHF35, desmin, and for the striated muscular markers myoglobin, sarcomeric actin (Fig 3), MyoD1, and MyF3. In some cells, striations and Zband material were also evident with electron microscopy. 


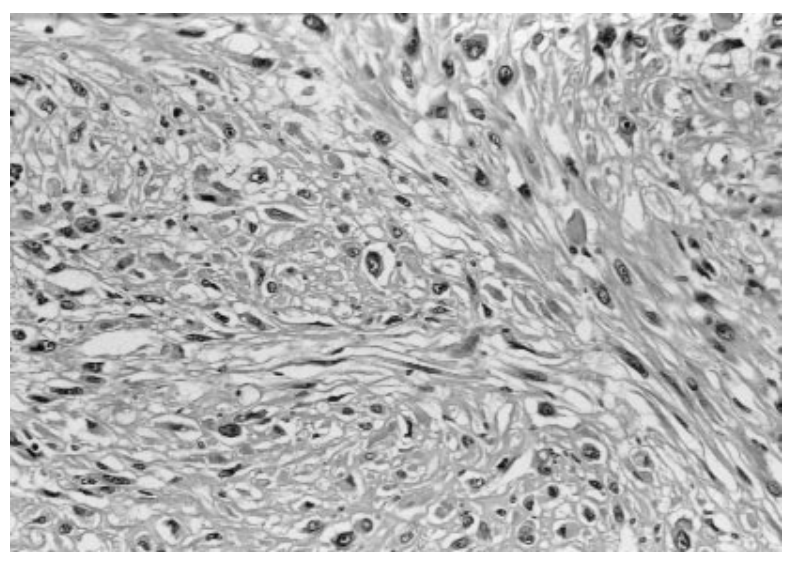

Fig 2. Pleomorphic sarcoma: spindle and neoplastic cells. Peripheral cytoplasmic vacuolization gives a spider-web shape to some neoplastic cells. (Hematoxylin-eosin stain; original magnification $\times 200$.)

Staging of the tumor while the patient was hospitalized failed to reveal other metastases and mediastinal diffusion. The patient refused to undergo further surgery or treatment. After 4 months, because of a worsening of clinical status, the patient was readmitted to the hospital. Dynamic computed axial tomography revealed a pleural effusion in the left part of the chest and some highly vascularized, solid masses in the anterior mediastinum and in the space between the aorta and the pulmonary artery. A skeletal scintiscan and an abdominal computed tomographic scan revealed bone and hepatic metastases, respectively. The patient underwent external beam radiation and chemotherapy with an improvement of the clinical status. Ten months after the operation, the patient is still alive, with disease.

Discussion. Primary sarcomas of the great vessels are rare. When they do occur, the most frequent site is the pulmonary artery, followed by the inferior vena cava. The aorta is a most uncommon site of sarcomas, which mainly involve the abdominal tract. ${ }^{1}$

According to their site of origin, aortic sarcomas have been divided into intimal and mural types. Mural type sarcomas may arise in the media or adventitia and usually have a less aggressive, although poor, clinical course. Intimal type sarcomas are characterized by a more aggressive behavior, leading to death within months; they may form plaques along the aortic intima, or they may grow as polyploid intraluminal vegetations. ${ }^{1}$ Intimal sarcomas are usually described as undifferentiated, pleomorphic sarcomas. In some reports, more specific histotypes have been identified, such as angiosarcoma, leiomyosarcoma, (myo)fibrosarcoma, and fibro(myxo) sarcoma. ${ }^{2}$ To our knowledge, lesions of the aorta with signs of rhabdomyosarcomatous differentiation have not been described to date. Rhabdomyosarcomas have been described in the pulmonary artery, ${ }^{3}$ and an origin from pluripotent precursor cells of the bulbus cordis have been suggested; a similar origin might be hypothesized in the aorta.

Because of their rarity, aortic sarcomas are not always suspected in the preoperative period. The initial clinical presenta-

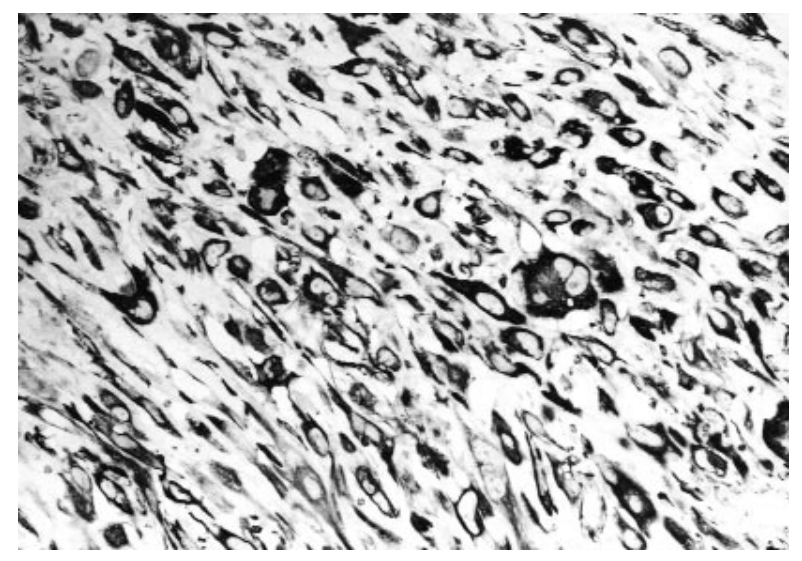

Fig 3. Many tumor cells strongly immunoreact with the striated muscle marker sarcomeric actin. (Alkaline phosphatase anti-alkaline phosphatase; original magnification $\times 200$.)

tion of aortic sarcomas may be nonspecific, with fever, weight loss, and anorexia. ${ }^{1}$ More frequently, they give rise to embolic phenomena. ${ }^{4}$ Chest pain, aortic occlusion, dissection, false aneurysm formation, and rupture have also been reported. ${ }^{1,5}$ In the presented case the lesion had the appearance of a saccular false aneurysm; the presence of calcifications on the aortic wall suggested a mechanism of complicated atherosclerotic plaque with contained rupture. The intraluminal polyploid formation, discovered at pathologic examination, was not visualized at contrast computed tomographic scan and during the operation, because of mural thrombus. Weakening of the aortic wall, by intramural extension of sarcoma, is likely to be the cause of the contained rupture. In the absence of a typical appearance of the lesion and of any of the symptoms of neoplasm, the possibility of a primary tumor of the aorta was not considered. However, this case gives the opportunity to underline that aortic tumors, being rare, have a nonspecific presentation; therefore routine intraoperative histologic examination of the retrieved specimen has to be performed in all patients who have symptoms of aortic disease.

\section{REFERENCES}

1. Burke AP, Virmani R. Sarcomas of the great vessels: a clinicopathological study. Cancer 1993;71:1761-73.

2. Haber LM, Truong L. Immunohistochemical demonstration of the endothelial nature of aortic intimal sarcomas. Am J Surg Pathol 1988;12:798-802.

3. Bleisch VR, Kraus FT. Polyploid sarcoma of the pulmonary trunk: analysis of the literature and report of a case with leptomeric organelles and ultrastructural feature of rhabdomyosarcoma. Cancer 1980;46:314-24.

4. Higgins R, Posner MC, Moosa HH, Staley C, Pataki KI, Mendelow H. Mesenteric infarction secondary to tumor emboli from primary aortic sarcoma: guidelines for diagnosis and management. Cancer 1991;68:1622-7.

5. Steinberg JB, Johnson ER, Benda JA, Lanza LA. Primary leiomyosarcoma of the thoracic aorta presenting as a contained rupture. Ann Thorac Surg 1993;56:1387-9. 